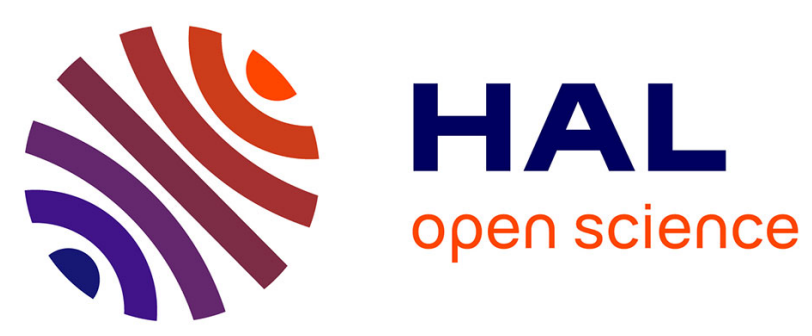

\title{
Buoyancy modelling with incompressible SPH for laminar and turbulent flows
}

\author{
Agnès Leroy, Damien Violeau, Martin Ferrand, Antoine Joly
}

\section{To cite this version:}

Agnès Leroy, Damien Violeau, Martin Ferrand, Antoine Joly. Buoyancy modelling with incompressible SPH for laminar and turbulent flows. International Journal for Numerical Methods in Fluids, 2015, 78 (8), pp.455 - 474. 10.1002/fld.4025 . hal-01557023

\section{HAL Id: hal-01557023 \\ https://hal.science/hal-01557023}

Submitted on 5 Jul 2017

HAL is a multi-disciplinary open access archive for the deposit and dissemination of scientific research documents, whether they are published or not. The documents may come from teaching and research institutions in France or abroad, or from public or private research centers.
L'archive ouverte pluridisciplinaire HAL, est destinée au dépôt et à la diffusion de documents scientifiques de niveau recherche, publiés ou non, émanant des établissements d'enseignement et de recherche français ou étrangers, des laboratoires publics ou privés. 


\title{
Buoyancy modelling with incompressible SPH for laminar and turbulent flows
}

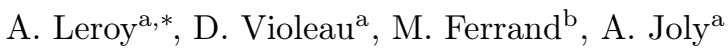 \\ ${ }^{a}$ Saint-Venant Laboratory for Hydraulics, Université Paris-Est (joint research unit EDF \\ RED, Cerema, ENPC), 6 quai Watier, 78400 Chatou, France \\ ${ }^{b}$ MFEE, EDF RESD, 6 quai Watier, 78400 Chatou, France
}

\begin{abstract}
This work aims at modelling buoyant, laminar or turbulent flows, using a 2D Incompressible Smoothed Particle Hydrodynamics (ISPH) model with accurate wall boundary conditions. The buoyancy effects are modelled through the Boussinesq approximation coupled to a heat equation, which makes it possible to apply an incompressible algorithm to compute the pressure field from a Poisson equation. Based on our previous work (Leroy et al., 2014), we extend the unified semi-analytical wall boundary conditions to the present model. The latter is also combined to a Reynolds-Averaged Navier-Stokes approach to treat turbulent flows. The $k-\epsilon$ turbulence model is used, where buoyancy is modelled through an additional term in the $k-\epsilon$ equations like in mesh-based methods. We propose a unified framework to prescribe isothermal (Dirichlet) or imposed heat flux (Neumann) wall boundary conditions in ISPH. To illustrate this, a theoretical case is presented (laminar heated Poiseuille flow), where excellent agreement with the theoretical solution is obtained. Several benchmark cases are then proposed: a lock-exchange flow, two laminar and one turbulent flow in differentially heated cavities, and finally a turbulent heated Poiseuille flow. Comparisons are provided with a Finite-Volume (FV) approach using an open-source industrial code.
\end{abstract}

Keywords: SPH, incompressible, boundary conditions, turbulence, buoyancy, temperature

\footnotetext{
*Corresponding author. tel: +33 (0)6 67889213

Email addresses: agnes.leroy@edf.fr (A. Leroy), damien.violeau@edf.fr (D. Violeau)
} 


\section{Introduction}

This work aims at modelling buoyant, laminar or turbulent flows, using a 2D Incompressible Smoothed Particle Hydrodynamics (ISPH) model where wall boundary conditions are imposed through an appropriate and accurate technique. The word 'buoyant' herein refers to flows where the density varies in space and time due to an active scalar like temperature or salinity. For simplicity, we will always refer to 'temperature' and denote the scalar field by $T$. Buoyancy plays an important part in many industrial and environmental flows. Modelling its effects through a Lagrangian method presents the advantage of avoiding artificial diffusion, since the advection is inherently represented by the particle displacement.

The buoyancy effects are modelled here through the Boussinesq approximation coupled to a heat equation, so that density variations only act through an additional term in the gravity force of the momentum equation. As a consequence, the density is considered constant in all governing equations, which makes it possible to apply an incompressible algorithm to compute the pressure field from a Poisson equation. This approach has been widely used in CFD (Computational Fluid Dynamics) with mesh-based methods (see e.g. [1] regarding the Finite Elements method or [2] with the Finite Volume method), but is still rather new in the SPH publications. To our knowledge, the only similar works so far have been proposed by Szewc et al. [3] and by Ghasemi et al. [4], with classical but rather crude approaches for prescribing wall boundary conditions (ghost particles and dummy particles respectively). Based on our previous work [5], we extend here the unified semi-analytical wall boundary conditions (hereinafter denoted USAW) to the present SPH model of buoyancy. This technique has proved its ability to work in the framework of ISPH. The main advantage of working with the USAW boundary conditions, besides their accuracy on complex wall geometries in arbitrary space dimension, is that they make it possible to exactly impose arbitrary wall boundary conditions on the temperature. Indeed, we propose a unified framework to prescribe isothermal (Dirichlet) or imposed heat flux (Neumann) wall boundary conditions in ISPH. As in [5], the proposed model is combined to a Reynolds-Averaged Navier-Stokes (RANS) approach to treat turbulent flows. The $k-\epsilon$ turbulence model is used, 
as it is today a standard approach for industrial and environmental flows. The second novelty proposed here is to apply the latter model to SPH with buoyancy. This is achieved by means of usual tools for grid-based methods, i.e. introducing a buoyant term in the $k-\epsilon$ equations (see e.g. [6]). The latter term is proportional to the temperature gradient, here computed with the SPH tools. Again, the USAW technique is used to compute the relevant boundary terms. To illustrate the ability of the present model to predict buoyant flows, a theoretical case is presented (laminar heated Poiseuille flow), where excellent agreement with the theoretical solution is obtained. Several benchmark cases are then proposed: a lock-exchange flow, two laminar and one turbulent flow in differentially heated cavities, and finally a turbulent heated Poiseuille flow. Comparisons are provided with a Finite-Volume (FV) approach using an open-source industrial code.

\section{Governing equations and modelling choices}

The system of equations to be solved is composed of the incompressible Reynolds-Averaged Navier-Stokes (RANS) equations coupled to a heat equation and to the $k-\epsilon$ turbulence closure. As explained in the Introduction, the formalism of heat transfer was chosen, but the reasoning applies to other active scalars like salinity. The Boussinesq approximation is used to account for density variations so that the system reads:

$$
\left\{\begin{array}{l}
\boldsymbol{\nabla} \cdot \boldsymbol{v}=0 \\
\frac{d k}{d t}=\mathbb{P}+\mathbb{G}-\epsilon+\frac{1}{\rho} \nabla \cdot\left(\mu_{k} \boldsymbol{\nabla} k\right) \\
\frac{d \epsilon}{d t}=\frac{\epsilon}{k}\left(C_{\epsilon_{1}} \mathbb{P}+C_{\epsilon_{3}} \mathbb{G}-C_{\epsilon_{2}} \epsilon\right)+\frac{1}{\rho} \nabla \cdot\left(\mu_{\epsilon} \boldsymbol{\nabla} \epsilon\right) \\
\frac{d \boldsymbol{v}}{d t}=-\frac{1}{\rho} \nabla \tilde{p}+\frac{1}{\rho} \boldsymbol{\nabla} \cdot\left(\mu_{E} \boldsymbol{\nabla} \boldsymbol{u}\right)+\boldsymbol{g}\left(1-\beta\left(T-T_{0}\right)\right) \\
\frac{d \boldsymbol{r}}{d t}=\boldsymbol{v} \\
\frac{d T}{d t}=K_{E} \nabla^{2} T
\end{array}\right.
$$

In this system, $\boldsymbol{r}$ is the particle position, $\boldsymbol{v}$ is the Lagrangian velocity and $\boldsymbol{u}$ is the Eulerian velocity. Both velocities are equal in our SPH model except for wall boundary particles as we will see in Section 3.3 . $t$ is the time, $\rho$ is the 
density (which remains constant due to the Boussinesq approximation), $k$ is the turbulent kinetic energy field, $\epsilon$ is its dissipation rate, $\mu$ is the dynamic molecular viscosity (which may be a function of the temperature), $\mu_{T}$ is the dynamic eddy viscosity, $\mu_{E}=\mu+\mu_{T}$ is the effective (i.e. total) dynamic viscosity. We also define the two variables $\mu_{k}=\mu+\frac{\mu_{T}}{\sigma_{k}}$ and $\mu_{\epsilon}=\mu+\frac{\mu_{T}}{\sigma_{\epsilon}} . \sigma_{k}, C_{\epsilon_{1}}, C_{\epsilon_{2}}$ and $\sigma_{\epsilon}$ are model constants described in Table [1. $\mathbb{P}$ is the production of turbulent kinetic energy and $\mathbb{G}$ is a buoyancy production/destruction term. $C_{\epsilon 3}$ is set to 1 if $\mathbb{G} \leq 0$ and 0 otherwise. $\mathbb{P}$ is calculated according to a mixed linear-quadratic model [7]:

$$
\mathbb{P}=\min \left(\sqrt{C_{\mu}} k S, \nu_{T} S^{2}\right)
$$

where $S=\sqrt{2 \boldsymbol{S}: S}$ is the scalar mean rate-of-strain. $\mathbb{G}$ is a buoyancy production/destruction term calculated as [6]:

$$
\mathbb{G}=\beta K_{T} \nabla T \cdot \boldsymbol{g}
$$

$\boldsymbol{g}$ is the gravity field (of magnitude $g=9.81 \mathrm{~m}^{2} \mathrm{~s}^{-1}$ ), $\tilde{p}=p+\frac{2}{3} k$ with $p$ the pressure, $\beta$ is the coefficient of thermal expansion, $T$ is the temperature field, $T_{0}$ is the mean temperature. $K_{E}=K+K_{T}$ is the effective thermal diffusivity, with $K$ the molecular thermal diffusivity and $K_{T}=\frac{\nu_{T}}{P r_{T}}, P r_{T}$ being the turbulent Prandtl number, taken as 0.85 (see e.g. [6]). We also define $\nu=\frac{\mu}{\rho}$ and $\nu_{T}=\frac{\mu_{T}}{\rho}$. $\nu_{T}$ is calculated as a function of $k$ and of $\epsilon$ as usual [8]:

$$
\nu_{T}=C_{\mu} \frac{k^{2}}{\epsilon}
$$

where $C_{\mu}$ is a constant defined in Table .1 . Note that the eddy viscosity is imposed equal to zero at the walls. In case of a laminar flow, $\nu_{T}$ is set to zero and the $k$ and $\epsilon$ equations are not solved.

\section{Buoyancy modelling with incompressible SPH and the USAW bound- ary conditions}

\subsection{Space discretisation}

In all this paper we work in 2D. We assume the reader is familiar with the standard SPH method (see 9] for more details). With the SPH method and the USAW boundary conditions the space discretisation is done through different sets of particles and boundary elements called segments. Fluid particles which 
do not belong to a boundary are called free particles $a \in F$, whereas particles belonging to a boundary are called vertex particles $v \in V$. The latter are linked together by segments $s \in S$, which compose a mesh of the boundary [10, 5] (see Figure 11. The set of all fluid particles is noted $P=F \cup V$. In this framework, the discrete SPH interpolation of a field $A$ at particle $a$ with position $\boldsymbol{r}_{a}$ reads:

$$
[A]_{a}^{\gamma}=\frac{1}{\gamma_{a}} \sum_{b \in P} V_{b} A_{b} w_{a b}
$$

where $V_{b}=\frac{m_{b}}{\rho}$ is the volume of particle $b, m_{b}$ being its mass, and $w_{a b}=$ $w\left(\boldsymbol{r}_{a}-\boldsymbol{r}_{b}\right), w$ being the SPH kernel. In general the value of a field $A$ at a particle $b$ is denoted by $A_{b} . \gamma_{a}$ is the wall renormalisation factor, defined as in [11, 10]:

$$
\gamma_{a}=\int_{\Omega \cap \Omega_{a}} w\left(\boldsymbol{r}_{a}-\boldsymbol{r}^{\prime}\right) d^{n} \boldsymbol{r}^{\prime}
$$

where $\Omega$ is the fluid domain, $\Omega_{a}$ is the compact support of the kernel at particle $a$ and $n$ is the space dimension. $\gamma_{a}$ is computed according to an analytical formula [5].

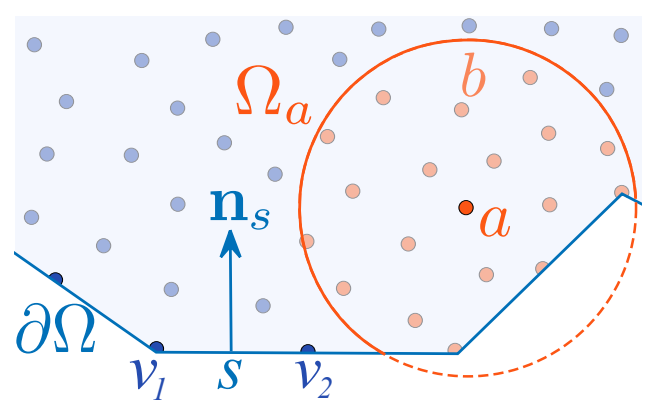

Figure 1: Sketch of the different entities involved in the space discretisation with SPH and USAW wall boundary conditions.

We define two SPH gradient operators of the field $A$ which will be used for different purposes:

$$
\begin{gathered}
\mathbf{G}_{a}^{\gamma,+}\left\{A_{b}\right\}=\frac{1}{\gamma_{a}} \sum_{b \in P} V_{b}\left(A_{a}+A_{b}\right) \boldsymbol{\nabla} w_{a b}-\frac{1}{\gamma_{a}} \sum_{s \in S}\left(A_{a}+A_{s}\right) \boldsymbol{\nabla} \gamma_{a s} \\
\mathbf{G}_{a}^{\gamma,-}\left\{A_{b}\right\}=-\frac{1}{\gamma_{a}} \sum_{b \in P} V_{b} A_{a b} \boldsymbol{\nabla} w_{a b}+\frac{1}{\gamma_{a}} \sum_{s \in S} A_{a s} \boldsymbol{\nabla} \gamma_{a s}
\end{gathered}
$$


where $A_{a b}=A_{a}-A_{b}, A_{a s}=A_{a}-A_{s}$ and $\nabla \gamma_{a s}$ is the contribution of segment $s$ to the gradient of $\gamma_{a}$, defined as:

$$
\boldsymbol{\nabla} \gamma_{a s}=\int_{\partial \Omega_{s} \cup \Omega_{a}} w\left(\boldsymbol{r}_{a}-\boldsymbol{r}^{\prime}\right) \boldsymbol{n}_{s} d^{n-1} \boldsymbol{r}^{\prime}
$$

In this formula, $\partial \Omega_{s}$ is the boundary area spanned by segment $s$ and $\boldsymbol{n}_{s}$ is the inward unit normal to the wall at segment $s$ (Figure 1). $\nabla \gamma_{a s}$ is computed according to an analytical formula [10]. An SPH divergence of a field $\boldsymbol{A}$ is defined as:

$$
D_{a}^{\gamma}\left\{\boldsymbol{A}_{b}\right\}=-\frac{1}{\gamma_{a}} \sum_{b \in P} V_{b} \boldsymbol{A}_{a b} \cdot \boldsymbol{\nabla} w_{a b}+\frac{1}{\gamma_{a}} \sum_{s \in S} \boldsymbol{A}_{a s} \cdot \boldsymbol{\nabla} \gamma_{a s}
$$

The SPH Laplacian operator used in this work is the one proposed by Ferrand et al. [10] which reads:

$$
\begin{aligned}
L_{a}^{\gamma}\left\{B_{b}, A_{b}\right\} & =\frac{1}{\gamma_{a}} \sum_{b \in P} V_{b}\left(B_{a}+B_{b}\right) \frac{A_{a b}}{r_{a b}^{2}} \boldsymbol{r}_{a b} \cdot \nabla w_{a b} \\
& -\frac{1}{\gamma_{a}} \sum_{s \in S}\left[B_{s}(\nabla A)_{s}+B_{a}(\nabla A)_{a}\right] \cdot \nabla \gamma_{a s}
\end{aligned}
$$

where $B$ is a (variable) diffusion coefficient for the field $A$ and $r_{a b}=\left|\boldsymbol{r}_{a b}\right|$. In case $\boldsymbol{A}$ is a vector, the Laplacian will be noted $\mathbf{L}_{a}^{\gamma}\left\{B_{b}, \boldsymbol{A}_{b}\right\}$ and in case $B=1$ the Laplacian will be noted $L_{a}^{\gamma}\left\{A_{b}\right\}$. Later on, we will sometimes use the following notation for the boundary term of (11):

$$
L_{a}^{\text {bound }}\left\{B_{b}, A_{b}\right\}=-\frac{1}{\gamma_{a}} \sum_{s \in S}\left[B_{s}(\nabla A)_{s}+B_{a}(\nabla A)_{a}\right] \cdot \nabla \gamma_{a s}
$$

\subsection{Time discretisation}

In the turbulent case, $k_{a}$ and $\epsilon_{a}$ are calculated at the beginning of the iteration through a semi-implicit time-scheme making up the SPH form of the standard buoyant $k-\epsilon$ model (second and third lines of (1)):

$$
\begin{gathered}
\frac{k_{a}^{n+1}-k_{a}^{n}}{\delta t}=\mathbb{P}_{a}^{n}+\mathbb{G}_{a}-\epsilon_{a}^{n} \frac{k_{a}^{n+1}}{k_{a}^{n}}+\frac{1}{\rho} L_{a}^{\gamma}\left\{\mu_{k, b}, k_{b}^{n}\right\} \\
\frac{\epsilon_{a}^{n+1}-\epsilon_{a}^{n}}{\delta t}=\frac{\epsilon_{a}^{n}}{k_{a}^{n}}\left(C_{\epsilon_{1}} \mathbb{P}_{a}^{n}+C_{\epsilon_{3}} \mathbb{G}_{a}-C_{\epsilon_{2}} \epsilon_{a}^{n+1}\right)+\frac{1}{\rho} L_{a}^{\gamma}\left\{\mu_{\epsilon, b}, \epsilon_{b}^{n}\right\}
\end{gathered}
$$

where the superscript $n$ represents the iteration number and $\delta t$ is the time step. In $(13)$ and $(14)$ the dissipative terms are treated implicitly in order to avoid negative values of $k$ and $\epsilon$ (recall $\rho$ is a constant, so we omit the particle subscript 
for this variable). $\mathbb{P}_{a}$ is calculated according to (2), where $\boldsymbol{S}_{a}$ is computed with the following SPH approximation of the mean rate-of-strain tensor:

$$
\boldsymbol{S}_{a}=\frac{1}{2}\left[\mathbf{G}_{a}^{\gamma,-}\left\{\boldsymbol{u}_{b}\right\}+\left(\mathbf{G}_{a}^{\gamma,-}\left\{\boldsymbol{u}_{b}\right\}\right)^{T}\right]
$$

According to (3), $\mathbb{G}_{a}$ is modelled by:

$$
\mathbb{G}_{a}= \begin{cases}\frac{\beta C_{\mu}}{P r_{T}} \frac{k_{a}^{n} k_{a}^{n+1}}{\epsilon_{a}^{n}} \mathbf{G}_{a}^{\gamma,-}\left\{T_{b}\right\} \cdot \boldsymbol{g} & \text { if } \mathbf{G}_{a}^{\gamma,-}\left\{T_{b}\right\} \cdot \boldsymbol{g} \leq 0 \\ \frac{\beta C_{\mu}}{\operatorname{Pr}_{T}} \frac{\left(k_{a}^{n}\right)^{2}}{\epsilon_{a}^{n}} \mathbf{G}_{a}^{\gamma,-}\left\{T_{b}\right\} \cdot \boldsymbol{g} & \text { otherwise }\end{cases}
$$

Again, $\mathbb{G}_{a}$ is semi-implicited in case it is negative in order to avoid negative values of $k$ and $\epsilon$. After the computation of $k, \epsilon$ and $\nu_{T}$ (using (4)), the spacetime discretisation of (1) follows an ISPH predictor-corrector scheme with a pressure Poisson equation, as in [5], with an additional SPH diffusion equation for the temperature:

$$
\left\{\begin{array}{l}
\frac{\boldsymbol{v}_{a}^{*}-\boldsymbol{v}_{a}^{n}}{\delta t}=\frac{1}{\rho} \mathbf{L}_{a}^{\gamma}\left\{\mu_{E, b}, \boldsymbol{u}_{b}^{n}\right\}-\boldsymbol{g} \beta\left(T_{a}^{n}-T_{0}\right) \\
L_{a}^{\gamma}\left\{\tilde{p}_{b}^{n+1}\right\}=\frac{\rho}{\delta t} D_{a}^{\gamma}\left\{\boldsymbol{v}_{b}^{*}\right\} \\
\frac{\boldsymbol{v}_{a}^{n+1}-\boldsymbol{v}_{a}^{*}}{\delta t}=-\frac{1}{\rho} \mathbf{G}_{a}^{\gamma,+}\left\{\tilde{p}_{b}^{* n+1}\right\} \\
\boldsymbol{r}_{a}^{n+1 / 2}=\boldsymbol{r}_{a}^{n}+\frac{\delta t}{2} \boldsymbol{v}_{a}^{n+1} \\
\frac{T_{a}^{n+1}-T_{a}^{n}}{\delta t}=L_{a}^{\gamma}\left\{K_{E, b}, T_{b}^{n}\right\} \\
\tilde{\boldsymbol{r}}_{a}^{n+1 / 2}=\boldsymbol{r}_{a}^{n+1 / 2}+\delta \boldsymbol{r}_{a} \\
\tilde{\boldsymbol{v}}_{a}^{n+1}=\boldsymbol{v}_{a}^{n+1}+\mathbf{G}_{a}^{\gamma,-}\left\{\boldsymbol{v}_{b}^{n+1}\right\} \cdot \delta \boldsymbol{r}_{a} \\
\boldsymbol{r}_{a}^{n+1}=\tilde{\boldsymbol{r}}_{a}^{n+1 / 2}+\frac{\delta t}{2} \tilde{\boldsymbol{v}}_{a}^{n+1}
\end{array}\right.
$$

where $\boldsymbol{v}^{*}$ is the predicted velocity field, $\tilde{p}^{*}=\tilde{p}+\rho g z$ is the dynamic pressure and $\delta \boldsymbol{r}_{a}$ is a particle shifting defined as in [5]:

$$
\delta \boldsymbol{r}_{a}=-0.35 h^{2} \mathbf{G}_{a}^{\gamma,+}\{1\}
$$

The particle shifting has been used to modify the velocity through a first-order Taylor expansion (7th line of (17)). The fields $\boldsymbol{u}, k, \epsilon$ and $T$ are corrected the same way.

The last three steps of (17) correspond to a stabilising procedure (see [12, [5]). Note that system (17) was written here for confined flows since all the validation 
cases considered in this paper are confined. This is why the resolution is done with the dynamic pressure instead of the total pressure, which yields more accurate results. In case of free-surface flows this can not be done because of the pressure Dirichlet condition on the free-surface, so that the term involving gravity in the first line of 17 becomes $-\boldsymbol{g}\left(\beta\left(T_{a}^{n}-T_{0}\right)-1\right)$ and $\tilde{p}^{*}$ is replaced by $\tilde{p}$ in the second and third lines of (17).

\subsection{Imposition of wall boundary conditions on $\boldsymbol{u}, \boldsymbol{v}, k$ and $\epsilon$}

The present section summarises our wall boundary conditions. Our technique is based on an analogy with Finite Volume (FV) and was validated in [5], especially for the $k-\epsilon$ turbulent model. The imposition of pressure wall boundary conditions is done as in [5] and is not described in this paper. A Dirichlet boundary condition is imposed on $\boldsymbol{v}$ : the Lagrangian velocity of the walls is imposed so that the model includes the treatment of forced wall movement through the velocities of the vertex. The velocities of the segments are then defined by:

$$
\boldsymbol{v}_{s}=\frac{1}{N_{s}} \sum_{V_{s}} \boldsymbol{v}_{v}
$$

where $V_{s}$ is the set of vertices linked to $s$ and $N_{s}$ its size (in $2 \mathrm{D}, N_{s}=2$ ). Non-homogeneous Neumann boundary conditions are imposed on $\boldsymbol{u}, k$ and $\epsilon$. In such cases, the prescription of wall boundary conditions is done by imposing both the flux and the value of the field at the wall. Therefore, the compatibility of the fields values and fluxes at the wall must be ensured. This holds for the temperature field as well (see section 3.4). The Neumann wall boundary conditions are applied through the surface term of the Laplacian operator 12 like in mesh-based methods, whereas the Dirichlet boundary conditions are imposed at the vertex particles which are involved in the summations over $b \in P$ in the Laplacian, gradient and divergence operators. Thus, in the aforementioned equations the particles $a$ belong to $F$. In the subsequent subsections, the notation $A_{a b}=A_{a}-A_{b}$ will be widely applied as in Section 3.1 .

\subsubsection{Wall boundary condition on $\boldsymbol{u}$}

A non-homogeneous Neumann condition is applied to the Eulerian velocity field: 


$$
\frac{1}{\rho} \mathbf{L}_{a}^{\text {surf }}\left\{\mu_{E, b}, \boldsymbol{u}_{b}\right\}=-\frac{2}{\gamma_{a} \rho} \sum_{s \in S} \mu_{E, a^{\prime}}\left(\frac{\partial \boldsymbol{u}}{\partial \boldsymbol{n}}\right)_{a^{\prime}} \cdot \boldsymbol{\nabla} \gamma_{a s}
$$

where $a^{\prime}$ is a fictitious point placed at $\frac{r_{a s}}{2}$. In the laminar case, the velocity distribution near the wall is almost linear, thus:

$$
\mu_{E, a^{\prime}}\left(\frac{\partial \boldsymbol{u}}{\partial \boldsymbol{n}}\right)_{a^{\prime}} \cdot \boldsymbol{n}_{s}=\mu \frac{\boldsymbol{v}_{a s} \cdot \boldsymbol{t}_{a s}}{\delta r_{a s}} \boldsymbol{t}_{a s}
$$

where

$$
\left\{\begin{array}{c}
\boldsymbol{t}_{a s}=\frac{\boldsymbol{v}_{a s}-\left(\boldsymbol{v}_{a s} \cdot \boldsymbol{n}_{s}\right)}{\left|\boldsymbol{v}_{a s}-\left(\boldsymbol{v}_{a s} \cdot \boldsymbol{n}_{s}\right)\right|} \\
\delta r_{a s}=\max \left(\boldsymbol{r}_{a s} \cdot \boldsymbol{n}_{s}, \delta r\right)
\end{array}\right.
$$

with $\delta r$ the initial interparticular space.

In the turbulent case, a two layers wall function is used for the velocity near the wall. The Neumann condition reads:

$$
\mu_{E, a^{\prime}}\left(\frac{\partial \boldsymbol{u}}{\partial \boldsymbol{n}}\right)_{a^{\prime}} \cdot \boldsymbol{n}_{s}=u_{*, a^{\prime}}^{2} \boldsymbol{t}_{a s}
$$

where $u_{*, a^{\prime}}$ is the friction velocity at the wall seen by particle $a$, which is a solution of:

$$
\begin{cases}\frac{\boldsymbol{v}_{a s} \cdot \boldsymbol{t}_{a s}}{u_{*, a^{\prime}}}=y_{a^{\prime}}^{+} & \text {if } y_{a^{\prime}}^{+} \leq y_{\text {lim }}^{+} \\ \frac{\boldsymbol{v}_{a s} \cdot \boldsymbol{t}_{a s}}{u_{*, a^{\prime}}}=\frac{1}{\kappa} \ln \left(\frac{\delta r_{a s} u_{*, a^{\prime}}}{\nu}\right)+5.2 & \text { if } y_{a^{\prime}}^{+}>y_{l i m}^{+}\end{cases}
$$

where $y_{a^{\prime}}^{+}=\frac{\delta r_{a s} u_{k, a^{\prime}}}{\nu}, y_{\text {lim }}^{+}=\frac{1}{\kappa}$ and $\kappa$ is the von Kármán constant (see Table 1 . The second line of $(24)$ is solved through an iterative process.

On the other hand, the velocity at the vertex particles is left to evolve according to the viscous term:

$$
\boldsymbol{u}_{v}^{n+1}=\boldsymbol{u}_{v}^{n}+\delta t \frac{1}{\rho} \mathbf{L}_{v}^{\gamma}\left\{\mu_{E, b}, \boldsymbol{u}_{b}\right\}
$$

but its normal component is imposed to be equal to zero by projecting $\boldsymbol{u}_{v}^{n+1}$ along the tangent to the wall.

\subsubsection{Wall boundary condition on $k$}

For the turbulent kinetic energy, a homogeneous Neumann condition is applied:

$$
L_{a}^{\text {bound }}\left\{\mu_{k, b}, k_{b}\right\}=0
$$


A compatible Dirichlet boundary condition on $k$ is imposed at all vertex particles $v$ :

$$
k_{v}=\frac{1}{N_{v}} \sum_{s \in S_{v}} k_{s}, k_{s}=\frac{1}{\alpha_{s}} \sum_{b \in F} V_{b} k_{b} w_{s b}
$$

where $S_{v}$ is the set of segments linked to $v, N_{v}$ is its size and $\alpha_{s}$ is the Shepard filter:

$$
\alpha_{s}=\sum_{b \in P} V_{b} w_{s b}
$$

\subsubsection{Wall boundary condition on $\epsilon$}

Here again, a non-homogeneous Neumann condition is applied according to the theory of turbulent boundary layer:

$$
L_{a}^{\text {bound }}\left\{\mu_{\epsilon, b}, \epsilon_{b}\right\}=\frac{4 C_{\mu}}{\sigma_{\epsilon} \gamma_{a}} \sum_{s \in S} \frac{k_{a}^{2}}{\delta r_{a s}}\left|\nabla \gamma_{a s}\right|
$$

A compatible Dirichlet boundary condition is imposed on $\epsilon$ at all vertex particles $v$ (see [5] for more detail):

$$
\epsilon_{v}=\frac{1}{N_{v}} \sum_{s \in S_{v}} \epsilon_{s}, \epsilon_{s}=\frac{1}{\alpha_{s}} \sum_{b \in F} V_{b}\left(\epsilon_{b}+\frac{4 C_{\mu}^{3 / 4} k_{b}^{3 / 2}}{\kappa \delta r_{s b}}\right) w_{s b}
$$

\subsection{Imposition of wall boundary conditions on the temperature}

We will now extend the ideas of Subsection 3.3 to the temperature. Again, what follows was inspired by the FV technique. Wall boundary conditions on the temperature may be of Neumann or Dirichlet type. In either case, their prescription is done by imposing both a heat flux and a temperature value at the wall. The compatibility of the temperature values and of the heat flux at the wall should be ensured, which is explained in the next two subsections. The boundary part of the diffusion term in the heat equation reads:

$$
L_{a}^{\text {bound }}\left\{K_{E, b}, T_{b}\right\}=-\frac{2}{\gamma_{a}} \sum_{s \in S} \boldsymbol{Q}_{s}^{T} \cdot \nabla \gamma_{a s}
$$

where $\boldsymbol{Q}_{s}^{T}=K\left(\frac{\partial T}{\partial \boldsymbol{n}}\right)_{s}$ is the heat flux at the wall (note that since $\nu_{T}=0$ at the wall, this also holds for $K_{T}$ ). The values of the heat flux and of the temperature at the wall depend on the type of wall boundary conditions. 


\subsubsection{Neumann wall boundary condition}

In this case the values of $\boldsymbol{Q}_{s}^{T}$ are imposed. A compatible Dirichlet condition is prescribed at the vertex particles, depending on the imposed flux:

$$
T_{v}=\frac{1}{N_{v}} \sum_{s \in S_{v}} T_{s}, T_{s}=\frac{1}{\alpha_{s}} \sum_{b \in F} V_{b}\left(T_{b}-\frac{\boldsymbol{Q}_{s}^{T} \cdot \boldsymbol{n}_{s}}{K} \delta r_{s b}\right) w_{s b}
$$

The previous choice for the temperature of the wall segments $T_{s}$ is inspired from the conditions described above for the $k-\epsilon$ model. It directly stems from a first-order Taylor expansion of the temperature near the wall.

\subsubsection{Dirichlet wall boundary condition}

In this case the value of the temperature is prescribed at the vertex particles. The $\boldsymbol{Q}_{s}^{T}$ are imposed in a consistent way with the Dirichlet condition, so that the boundary term 31 reads:

$$
L_{a}^{\text {bound }}\left\{K_{E, b}, T_{b}\right\}=-\frac{2}{\gamma_{a}} \sum_{s \in S} T_{*, a^{\prime}} u_{k, a^{\prime}}\left|\nabla \gamma_{a s}\right|
$$

where $u_{k, a^{\prime}}$ is a friction velocity (which should not be confused with $u_{*, a^{\prime}}$ defined above), and $T_{*, a^{\prime}}=\frac{\boldsymbol{Q}_{s}^{T} \cdot \boldsymbol{n}_{s}}{u_{k, a^{\prime}}}$ by definition. In laminar mode, a linear temperature wall function is applied:

$$
u_{k, a^{\prime}} T_{*, a^{\prime}}=\boldsymbol{Q}_{s}^{T} \cdot \boldsymbol{n}_{s}=K \frac{T_{a}-T_{s}}{\delta r_{a s}}
$$

with $T_{s}$ imposed through the Dirichlet condition, whereas in turbulent mode, $u_{k, a^{\prime}}$ is defined as:

$$
u_{k, a^{\prime}}=C_{\mu}^{1 / 4} k_{a}^{1 / 2}
$$

On the other hand, $T_{*, a^{\prime}}$ is defined as:

$$
T_{*, a^{\prime}}=\frac{T_{a}-T_{s}}{T_{a^{\prime}}^{+}}
$$

where $T_{a^{\prime}}^{+}$is computed through a three-layers wall function (see[13]):

$$
\begin{cases}T_{a^{\prime}}^{+}=\operatorname{Pr} y_{a^{\prime}}^{+} & \text {if } y_{a^{\prime}}^{+}<y_{1}^{+} \\ T_{a^{\prime}}^{+}=a_{2}-\frac{\operatorname{Pr}_{T}}{2 a_{1}\left(y_{a^{\prime}}^{+}\right)^{2}} & \text { if } y_{1}^{+} \leq y_{a^{\prime}}^{+}<y_{2}^{+} \\ T_{a^{\prime}}^{+}=\frac{\operatorname{Pr} T}{\kappa} \ln \left(y_{a^{\prime}}^{+}\right)+a_{3} & \text { if } y_{a^{\prime}}^{+}>y_{2}^{+}\end{cases}
$$


where $y_{a^{\prime}}^{+}$is defined as in (24), while

$$
\left\{\begin{aligned}
y_{1}^{+} & =\left(\frac{a_{4}}{P r}\right)^{1 / 3} \\
y_{2}^{+} & =\sqrt{\frac{a_{4} \kappa}{P r_{T}}} \\
a_{1} & =\frac{\operatorname{Pr}_{T}}{a_{4}} \\
a_{2} & =15 \operatorname{Pr}^{2 / 3} \\
a_{3} & =15 \operatorname{Pr}^{2 / 3}-\frac{\operatorname{Pr}_{T}}{2 \kappa}\left(1+\ln \left(\frac{a_{4} \kappa}{\operatorname{Pr}_{T}}\right)\right) \\
a_{4} & =1000
\end{aligned}\right.
$$

\section{Validation on laminar flows}

The 5th order Wendland kernel [14] was used for all the simulations with a smoothing length $h=2 \delta r$ (recall $\delta r$ is the initial interparticular spacing). In what follows the non-dimensional variables are denoted with a + superscript. Hereafter, the present buoyant incompressible SPH model with our boundary conditions will be referred to as ISPH-USAW. The FV results were obtained with the Code_Saturne open-source software [15].

\subsection{Laminar Poiseuille flow}

In order to check that the boundary conditions on the temperature are properly imposed by the method described in section 3.4 , two configurations of a $2 \mathrm{D}$ laminar plane Poiseuille flow were tested. A schematic description of their geometries is provided in Figure 2. Our coordinates are denoted $(x, z)$, the origin of the vertical axis being in the middle of the two walls, distant from $2 L$. In the first case (denoted TT), constant temperatures $T_{1}$ and $T_{2}$ were imposed on the lower and upper walls, respectively. In the second case (denoted QT), a constant heat flux $Q^{T}$ was imposed through the upper wall, while the lower wall remained isothermal at the temperature $T_{1}$. The flow, with bulk velocity $U$, is driven by a constant volumic force.

In the case TT the flow is governed by three dimensionless numbers:

$$
\left\{\begin{array}{l}
\operatorname{Pr}=\frac{\nu}{K} \\
\operatorname{Re}=\frac{U L}{\nu} \\
G r=\frac{\beta g \Delta T L^{3}}{\nu^{2}}
\end{array}\right.
$$




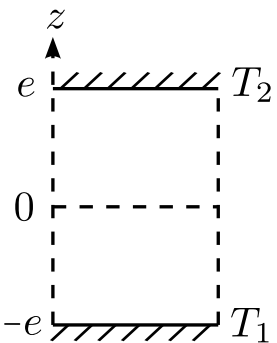

(a): TT

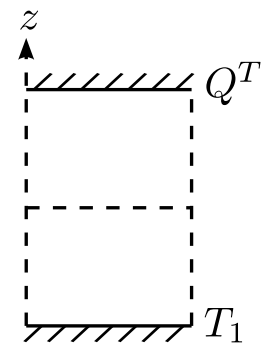

(b): QT

Figure 2: Laminar Poiseuille flow: sketch of the two configurations.

while in the case QT the flow is governed by four dimensionless numbers:

$$
\left\{\begin{array}{l}
\operatorname{Pr}=\frac{\nu}{K} \\
R e=\frac{U L}{\nu} \\
G r=\frac{\beta g \Delta T L^{3}}{\nu^{2}} \\
N u=\frac{L Q_{0}^{T}}{K \Delta T}
\end{array}\right.
$$

where $\Delta T=\max (T)-\min (T)$. In the two cases the Prandlt number was set to 1 and the Reynolds number to 50. In the QT case, the Nusselt number was set to 0.5 and the Grashoff number was set to 196, while in the TT case the Grashoff number was set to 98 .

The theoretical expressions of the dimensionless temperature and dynamic pressure $p^{*}=p+\rho g z$ for the two cases are presented in the Table.2 as functions of $R e, G r, N u$ and the dimensionless coordinates $x^{+}=\frac{x}{L}, z^{+}=\frac{z}{L}$. In both cases the velocity field is that of the ordinary Poiseuille flow. The dimensionless fields were defined as ${p^{*}}^{+}=\frac{p^{*}}{\rho \beta \Delta T g L}$ and $T^{+}=\frac{T-T_{1}}{\Delta T}$. The simulations were done with 902 particles $\left(\frac{\delta r}{L}=0.05\right)$. Figure 3 shows the vertical profiles of dimensionless temperature and dynamic pressure obtained with ISPH-USAW. It can be observed that an excellent agreement with the theory is obtained in both cases. In the case QT, the vertical variation of the fields does not depend on the Grashoff number. Thus, different values of the heat flux should yield the same results, which was checked with ISPH-USAW and gave similar errors between the model and the theory in all cases (with a Grashoff number up to 1960). With the chosen discretisation, the maximum relative error was of the order of $1 \%$, on the temperature and on the pressure. 

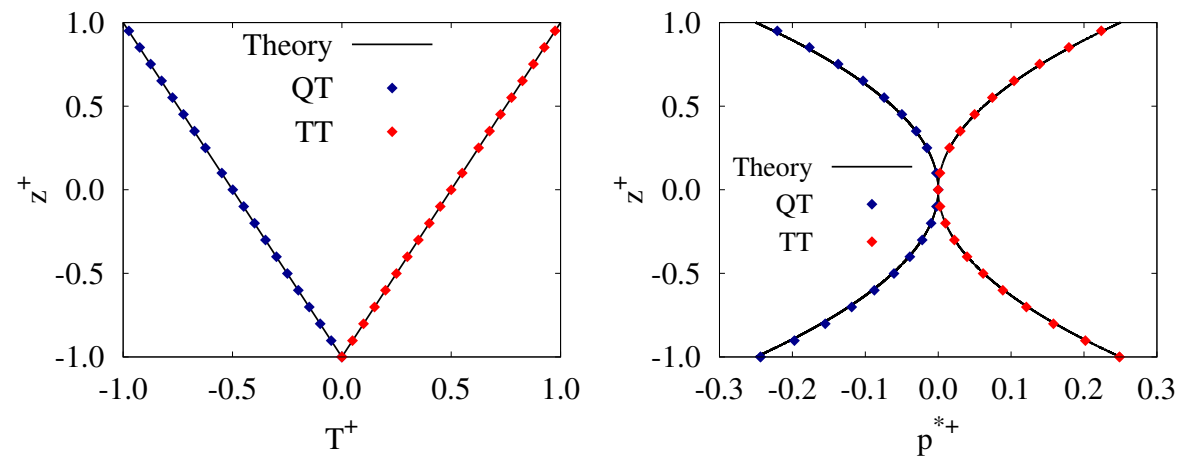

Figure 3: Laminar Poiseuille flow: profiles of dimensionless temperature (left) and dynamic pressure (right) on the vertical section of the channel obtained with ISPH-USAW, compared to the theoretical solutions of Table..2.

A convergence study was done for the case TT. The convergence plot is shown on the Figure 4, where the $L^{2}$ error on the temperature was computed through:

$$
L_{\text {error }}^{2}=\sqrt{\frac{1}{V_{\text {tot }}} \sum_{b \in P} V_{b}\left(\frac{T-T^{\text {theo }}}{T_{2}}\right)^{2}}
$$

where $T^{\text {theo }}$ is the theoretical temperature profile (linear profile) and $V_{\text {tot }}$ is the total volume of the particles $\left(V_{t o t}=\sum_{b \in P} V_{b}\right)$. The order of convergence is close to 2 for lower discretisations, and decreases for finer simulations because the discretisation error threshold is being reached. These results show that the imposition of Dirichlet and Neumann boundary conditions on the temperature is properly done with our method.

\subsection{Differentially heated square cavity}

The second validation case consists of a laminar flow in a differentially heated square cavity of size $L$ that was studied in [3] with the SPH method. The left and right walls are isothermal, the cold wall being on the right, and the upper and lower walls are adiabatic. The molecular Prandlt number is $\operatorname{Pr}=0.71$ and three values of the Rayleigh number $\operatorname{Ra}=\operatorname{Pr} \times G r$ were tested, namely $10^{3}, 10^{4}$ and $10^{5}$ (the Grashoff number is defined as in the 3rd line of 40 ). We define the dimensionless variables $x^{+}=\frac{x}{L}, z^{+}=\frac{z}{L}, \boldsymbol{u}^{+}=\frac{u L}{\nu}, T^{+}=\frac{T}{T_{h}}$ where $T_{h}$ is the temperature of the hot wall. A discretisation of $160 \times 160$ particles was used for the SPH simulations. Figure 5 shows the shape of the temperature and velocity 


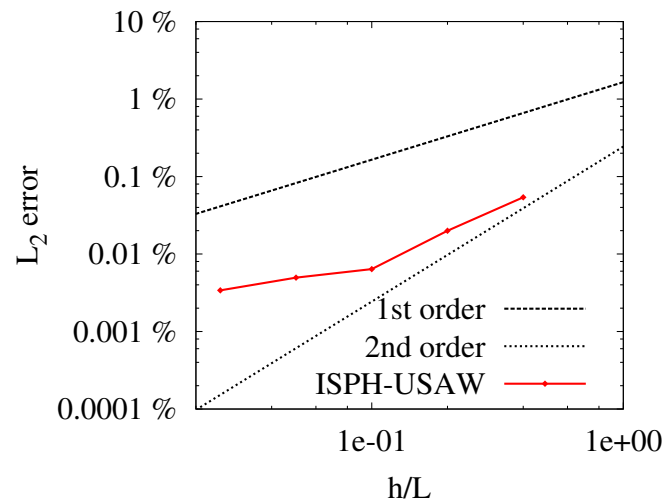

Figure 4: Laminar Poiseuille flow: convergence study concerning the error on the temperature field.

fields after convergence for $R a=10^{5}$. For the FV simulation, a discretisation of $512 \times 512$ cells was used. Figure 6 shows the dimensionless velocity and temperature profiles in $x^{+}=1 / 2$ and $z^{+}=1 / 2$ for $R a=10^{5}$. The SPH results are compared to FV and to the ones obtained by Wan et al. [16] by discrete singular convolution. Excellent agreement was obtained with both methods. The same quality of results was obtained with ISPH-USAW for $R a=10^{3}$ and $R a=10^{4}$.

The local Nusselt number measures the ratio of convective over conductive heat transfer across the boundary. For a wall segment $s$, it is defined as $N u_{s}=$ $L\left|\left(\frac{\partial T}{\partial \boldsymbol{n}}\right)_{s} \cdot \boldsymbol{n}_{s}\right| / \Delta_{T}$ and computed according to:

$$
N u_{s}=\frac{L}{\Delta_{T}}\left|\mathbf{G}_{s}^{\gamma,-}\left\{T_{b}\right\} \cdot \boldsymbol{n}_{s}\right|
$$

Figure 7 shows the evolution of the local Nusselt number along the cold wall for the three values of Rayleigh number. It appears that the behaviour is globally well predicted. However, a discrepancy occurs on the top of the curve $R a=$ $10^{5}$, which corresponds to the top right corner of the flow in Figure 5 where temperature gradients are rather high. It is a consequence of a lack of accuracy of the $\mathbf{G}^{\gamma,-}$ SPH gradient operator, used to compute the Nusselt number, since our temperature profiles are still in very good agreement with FV in this area.

\subsection{Differentially heated lid-driven cavity}

A differentially heated lid-driven cavity at $R e=\frac{U L}{\nu}=1000$ was tested, $L$ being the size of the cavity and $U$ the velocity of the lid. The flow is driven by 


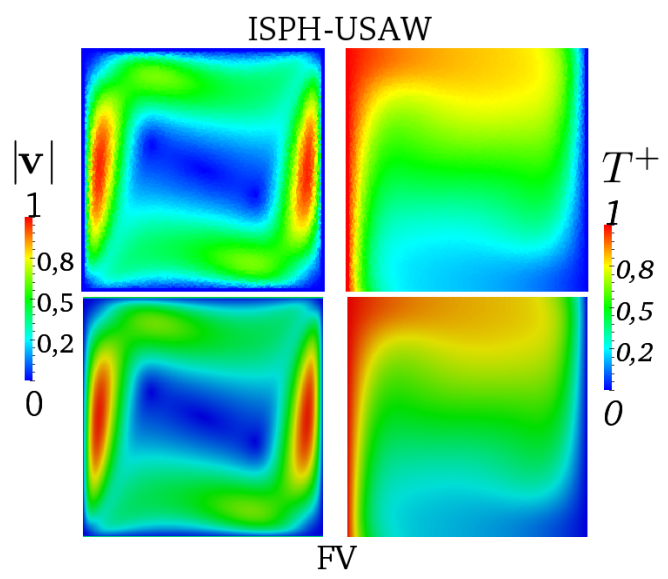

Figure 5: Differentially heated square cavity at $R a=10^{5}$. Shape of the temperature (right) and velocity (left) fields obtained with ISPH-USAW (top) and FV (bottom) after convergence.
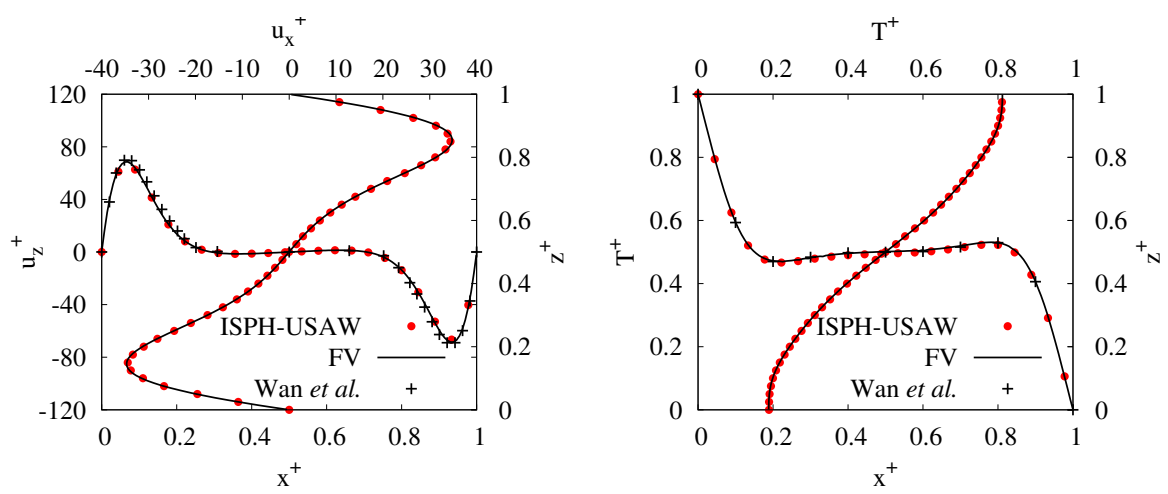

Figure 6: Differentially heated square cavity at $R a=10^{5}$. Profiles of velocity (left) and temperature (right) in $x^{+}=1 / 2$ and $z^{+}=1 / 2$ obtained with ISPHUSAW and FV after convergence. The horizontal profiles are also compared to the ones obtained by Wan et al. [16] with the discrete singular convolution method. 


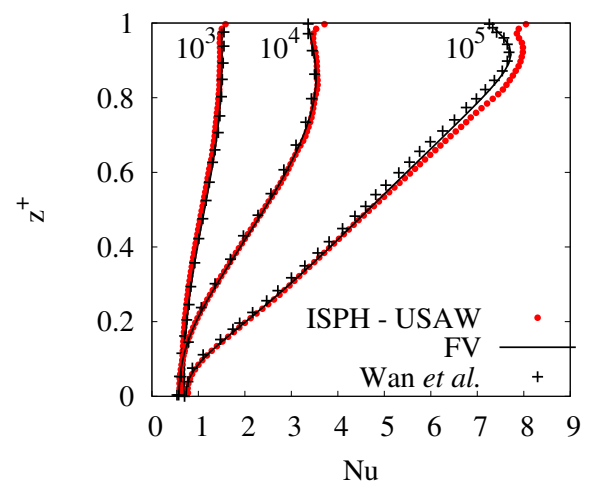

Figure 7: Differentially heated square cavity. Evolution of the Nusselt number along the cold wall of the cavity at $R a=10^{3}, 10^{4}$ and $10^{5}$. Comparison of the results obtained with ISPH-USAW, FV and discrete singular convolution [16] after convergence.

the shear force resulting from the lid motion and by the buoyancy force. The upper and lower walls are isothermal, their temperatures being of $T_{c}$ and $T_{h}$ respectively (with $T_{h}>T_{c}$ ). The molecular Prandlt number was set to 1 and the Grashoff number defined as in the previous section where $\Delta T=T_{h}-T_{c}$ ) to $10^{4}$. A discretisation of $160 \times 160$ particles was used for the SPH simulation. Figure 8 shows the shape of the temperature and velocity fields after convergence for a Rayleigh number of $10^{5}$. The results are compared to FV using a discretisation of $512 \times 512$ cells. We define the dimensionless variables $x^{+}=\frac{x}{L}, z^{+}=\frac{z}{L}$, $\boldsymbol{u}^{+}=\frac{\boldsymbol{u}}{U}, T^{+}=\frac{T}{T_{h}}$. Figures. 9 and 10 show the velocity and temperature profiles along $x^{+}=1 / 2$ and $z^{+}=1 / 2$. Satisfactory agreement was obtained with FV for the velocity, while the temperature shows a very nice agreement. The discrepancy in the velocity profile is due to the difference in the space discretisation, which is here significantly lower with the present SPH model. Figure 11 shows the repartition of local Nusselt number (computed according to 42 ) along the upper and lower walls of the cavity. As observed in the previous test-case, the formula 42 used to compute the local Nusselt number lacks accuracy for high temperature gradients (left part of the dotted curve in Figure 11, i.e. top left angle of the flow in Figure 8).

\subsection{Lock-exchange}

The fourth validation case consists of a symmetric lock-exchange flow in a rectangular cavity of height $2 L$ and width $30 L$. This case was studied in [4] 


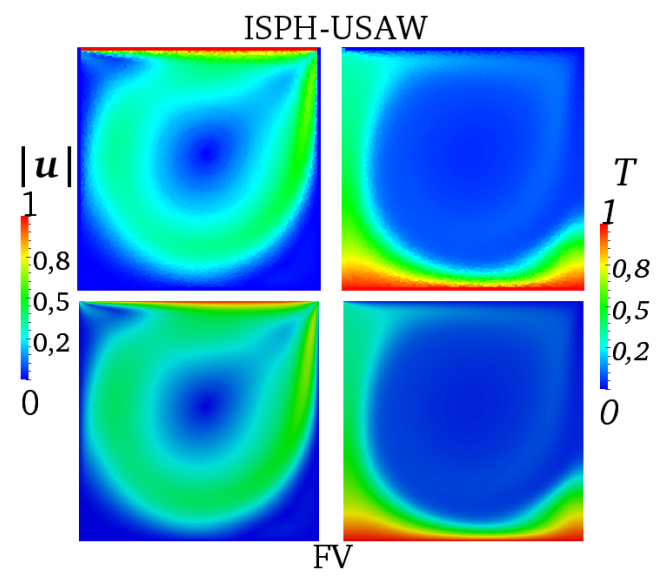

Figure 8: Differentially heated lid-driven cavity. Shape of the temperature (right) and velocity (left) fields obtained with ISPH-USAW (top) and FV (bottom) after convergence.

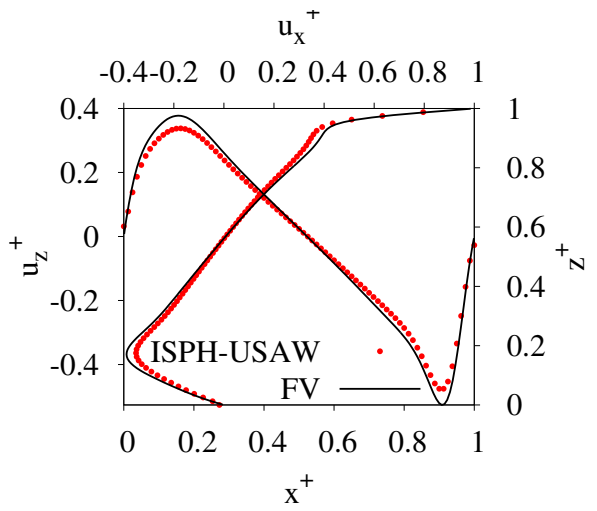

Figure 9: Differentially heated lid-driven cavity. Profiles of velocity in $x^{+}=1 / 2$ and $z^{+}=1 / 2$ obtained with ISPH-USAW and FV after convergence. 

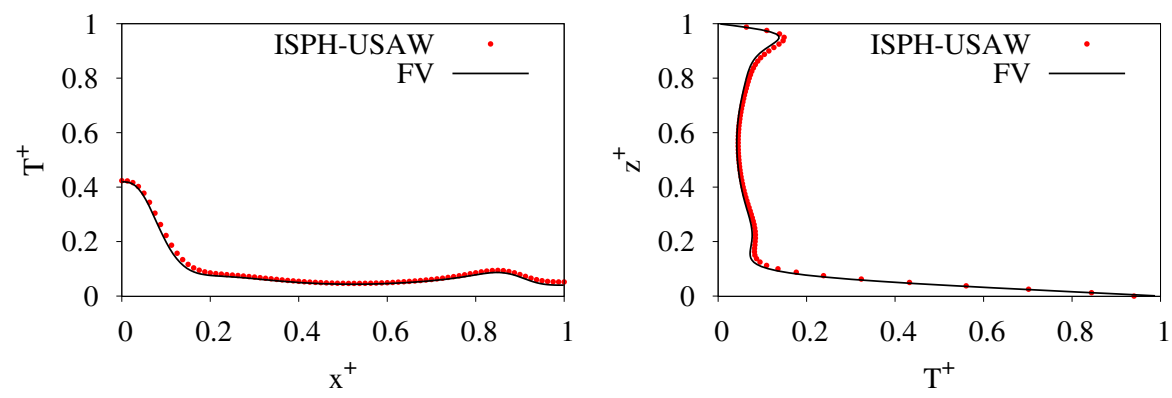

Figure 10: Differentially heated lid-driven cavity. Profiles of temperature in $z^{+}=1 / 2$ (left) and $x^{+}=1 / 2$ (right) obtained with ISPH-USAW and FV after convergence.

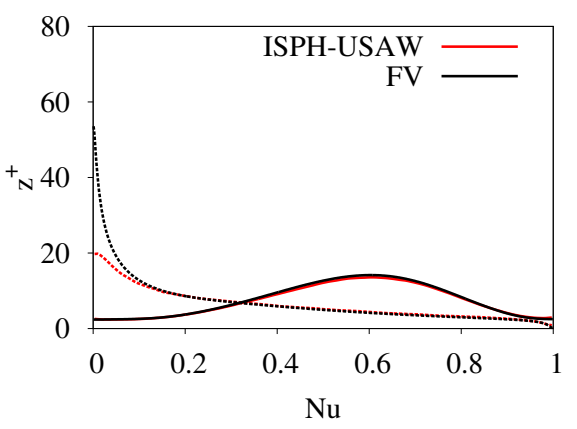

Figure 11: Differentially heated lid-driven cavity. Evolution of the Nusselt number along the lid (dash lines) and the lower wall (solid lines). Comparison of the results obtained with ISPH-USAW and FV after convergence. 


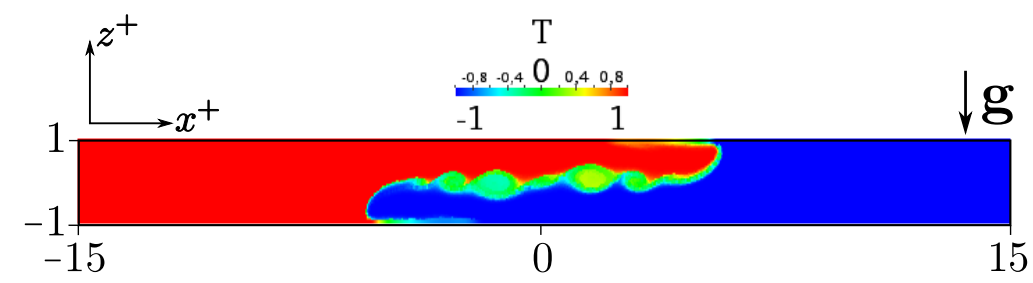

Figure 12: Lock-exchange: shape of the temperature field obtained with ISPHUSAW at $t^{+}=10$.

with the SPH method. All lengths are made dimensionless by $L$, the halfheight of the cavity. The flow consists of two fluids at temperatures $T_{h}$ (on the right) and $T_{c}$ (on the left) separated at $t=0$ at the half-width of the domain. The temperatures are made dimensionless by $T_{h}$ and the time by $\frac{L}{V}$ where $V=\sqrt{\beta \Delta T g L}$, with $\Delta T=T_{h}-T_{c}$. The dimensionless numbers describing the flow are $G r=1.25 \times 10^{6}$ and $\operatorname{Pr}=1$. Figure 12 shows the shape of the temperature field obtained with ISPH-USAW at $t^{+}=10$. The SPH simulation was done with a discretisation of $1500 \times 100$ particles. Figure 13 shows the temperature contours obtained with ISPH-USAW at several instants, compared to the ones obtained by Härtel et al. [17] through a 2-D Direct Numerical Simulation (DNS) with a mixed spectral/spectral-element discretisation in space together with finite differences in time. The shape and velocity of the front are well reproduced by the present SPH model. It should be noted that the results shown in Figure 13 were obtained with a symmetric operator for the pressure gradient (8), which better reproduced the vortices at the interface of the two fluids compared to the DNS results. Figure 14 shows the results obtained on this case with the antisymmetric SPH operator for the pressure gradient (7). The numerical diffusion is higher than with the symmetric operator and the instabilities do not develop as well. For all other test-cases in the present work, an antisymmetric operator (7) was used since it conserves linear momentum, but no significant differences were observed when using a symmetric operator.

\subsection{Infinite channel with a complex geometry}

This case was designed in order to show that the model is able to simulate flows with complex wall geometries. It consists of an infinite horizontal channel 


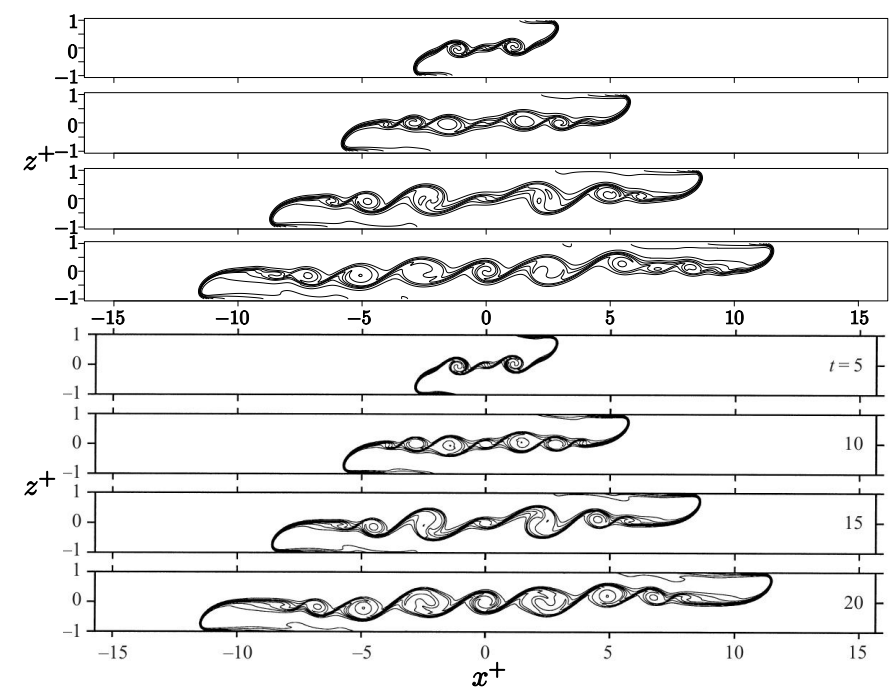

Figure 13: Lock-exchange: temperature contours at $t^{+}=5,10,15,20$. Top: ISPH-USAW. Bottom: Härtel et al. [17.

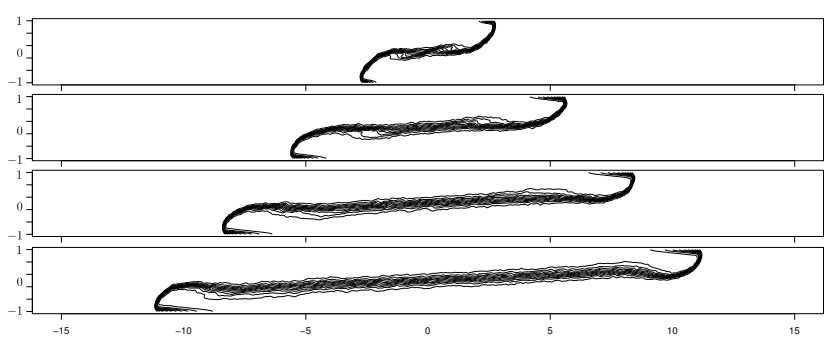

Figure 14: Lock-exchange: temperature contours at $t^{+}=5,10,15,20$ obtained with ISPH-USAW using an antisymmetric SPH gradient (7) for the pressure gradient. 


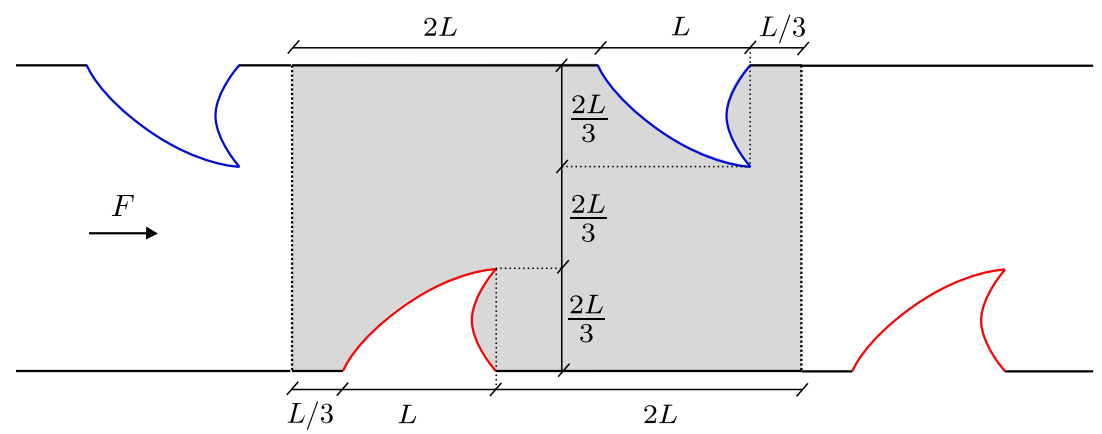

Figure 15: Infinite channel with a complex geometry: sketch of the case. The upper wedges are isothermal at the temperature $T_{c}$ (in blue) and the lower wedges are isothermal at the temperature $T_{h}$ (in red).

of height $2 L$ inside which the upper and lower walls present alternated circular wedges regularly spaced and isothermal at different temperatures. Al lengths are made dimensionless by $L$, the half-height of the channel. Figure 15 shows a sketch of the geometry. The flow is driven by a constant horizontal body force of magnitude $F=1 \mathrm{~ms}^{-2}$. The bulk velocity $U$ is then about equal to $2 m s^{-1}$ and the Reynolds number is $R e=20$. The Prandlt number is taken equal to 1 and the Grashoff number is taken equal to 981 . The lower wedges are isothermal at the temperature $T_{h}$ and the upper wedges are isothermal at the temperature $T_{c}$ (see Figure 15). The horizontal walls are adiabatic and periodic conditions are prescribed along the horizontal: only a portion of width $\frac{10 L}{3}$ of the domain is simulated (grey area on Figure 15. The Figure 16 shows surface plots of the velocity and temperature fields obtained with ISPH-USAW and FV after time convergence. The ISPH-USAW simulation counts 94941 particles while the FV simulation counts 113375 cells. The two models show very good qualitative agreement. Profiles of velocity and temperature were plotted after time convergence along the vertical lines $x^{+}=0.83, x^{+}=1.66, x^{+}=2.50$ and along the horizontal lines $z^{+}=0.33, z^{+}=1.00$ and $z^{+}=1.66$. The results are shown on the Figures 17 and 18 An excellent agreement between ISPH-USAW and FV is obtained, which shows that it is possible to accurately simulate non-isothermal flows with complex wall geometries with ISPH-USAW. 

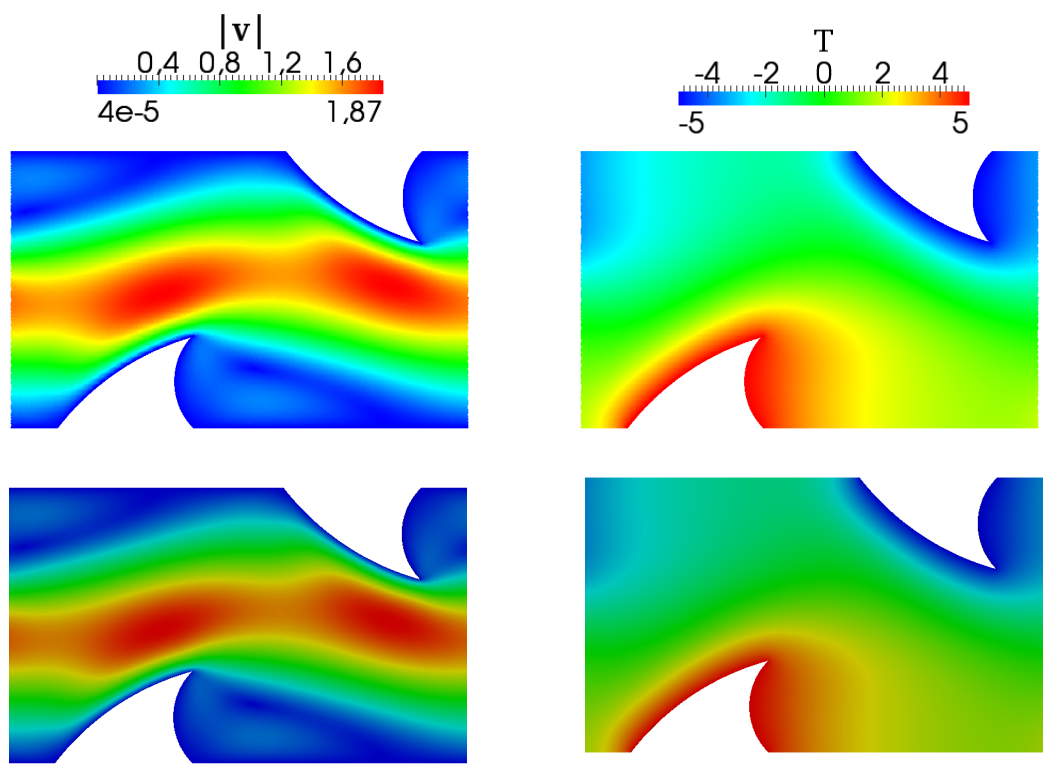

Figure 16: Infinite channel with a complex geometry: shape of the velocity (left) and temperature (right) fields obtained with ISPH-USAW (top) and FV (bottom) after time convergence.
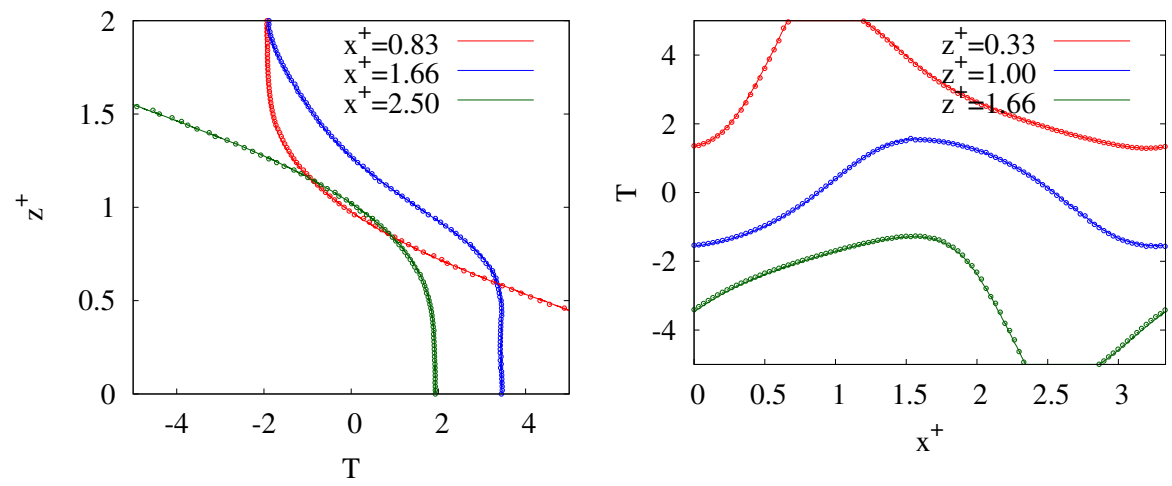

Figure 17: Infinite channel with a complex geometry. Profiles of temperature along $x^{+}=0.83,1.66,2.50$ (left) and $z^{+}=0.33,1.0,1.66$ (right) obtained with ISPH-USAW (solid lines) and FV (points) after convergence. 

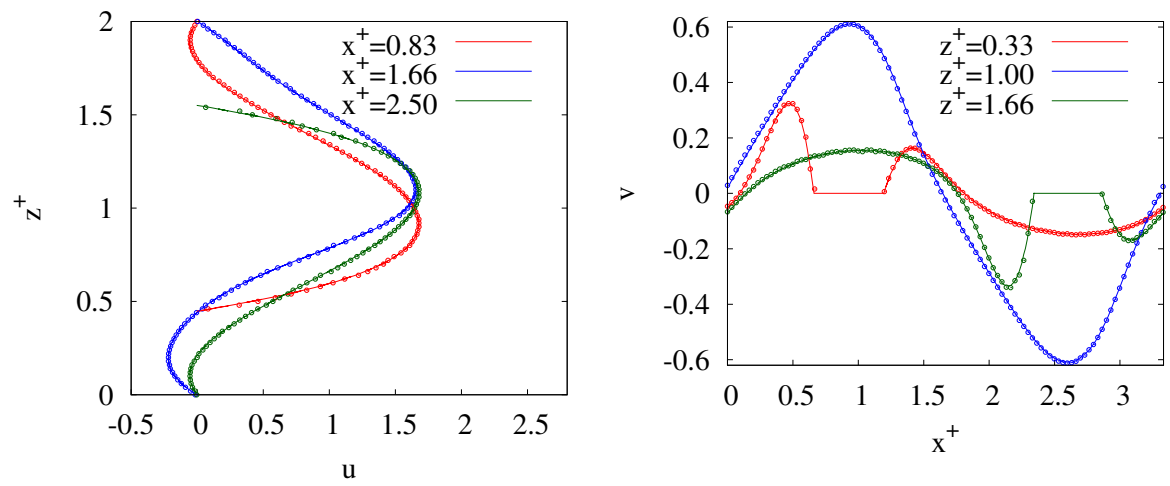

Figure 18: Infinite channel with a complex geometry. Profiles of horizontal velocity along $x^{+}=0.83,1.66,2.50$ (left) and vertical velocity along $z^{+}=$ 0.33, 1.0, 1.66 (right) obtained with ISPH-USAW (solid lines) and FV (points) after convergence.

\section{Validation on turbulent flows}

The validation of the $k-\epsilon$ turbulence model with buoyancy was done on two classical validation cases: a turbulent plane Poiseuille flow with two isothermal walls and a rectangular differentially heated cavity.

\subsection{Turbulent plane Poiseuille flow with two isothermal walls}

This case consists of a turbulent flow between two parallel, infinite vertical isothermal walls. The temperature of the left wall is set to $T_{h}$ and that of the right wall to $T_{c}$, with $T_{h}>T_{c}$. The flow is thus vertical, driven by a prescribed pressure gradient (the friction velocity is imposed) and by the temperature difference between the walls. The buoyancy force acts upward near the hot wall and downward near the cold wall. The friction Reynolds number, $R e_{*}=\frac{u_{*} L}{\nu}$ is set to 150 , where $L$ is the half-width of the channel and $u_{*}$ is the friction velocity, which was set to $1 \mathrm{~ms}^{-1}$ through the imposition of an upward volumic force:

$$
f=\frac{u_{*}^{2}}{L}-g \beta \frac{\sum_{b \in F} V_{b}\left(T_{b}-T_{0}\right)}{\sum_{b \in F} V_{b}}
$$

where $T_{0}=\frac{T_{h}+T_{c}}{2}$. The molecular Prandlt number is set to 0.71 and the Grashoff number to $G r=9.6 \times 10^{5}$. The following dimensionless variables are defined: $\boldsymbol{u}^{+}=\frac{u}{u_{*}}, x^{+}=\frac{x}{L}, T^{+}=\frac{T}{T_{h}}, k^{+}=\frac{k}{u_{*}^{2}}$ and $\epsilon^{+}=\frac{L \epsilon}{u_{*}^{3}}$. The results 

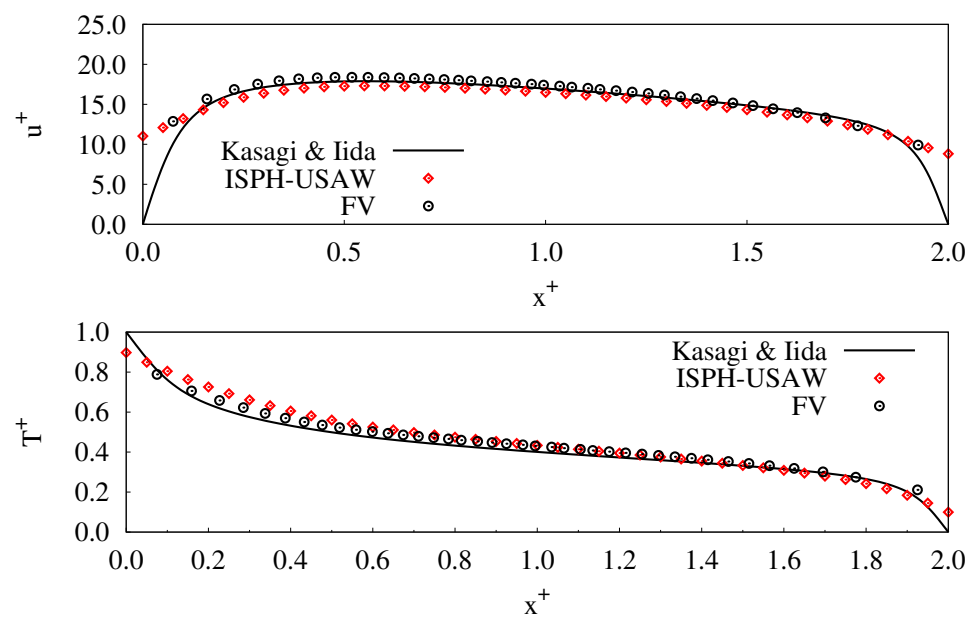

Figure 19: Turbulent plane Poiseuille flow. Profiles of velocity (top) and temperature (bottom) after convergence. Comparison of ISPH-USAW and FV with DNS results provided by Kasagi \& Iida [18].

obtained with SPH are compared to FV and to DNS data published by Kasagi \& Iida [18. Figures 19 and 20 show the profiles of velocity, temperature, turbulent kinetic energy and dissipation rate as functions of $x^{+}$. Good agreement is observed between the FV and $\mathrm{SPH}$ results, although some discrepancies on $k$ and $\epsilon$ are visible close to the walls. They seem to be due to the differences in the imposition of the wall boundary conditions on those fields between FV and ISPH-USAW.

\subsection{Differentially heated rectangular cavity in turbulent mode}

The last validation case consists of a differentially heated rectangular cavity of aspect ratio 4. The geometry of the case is described in Figure 21. The left and right walls are isothermal at temperature $T_{h}$ and $T_{c}$ respectively, with $T_{h}>T_{c}$. The upper and lower walls are adiabatic. The value of the molecular Prandtl number is 0.71 and that of the Rayleigh number $R a$ is $6.4 \times 10^{8}$. The lengths are made dimensionless by $4 L$ and the temperature by $\Delta T=T_{h}-T_{c}$. In the SPH simulation, a discretisation of $50 \times 200$ particles was used. The SPH results are compared to DNS results provided by Trias et al. [19] and to FV. For the FV simulation the same discretisation than in SPH was used. A comparison of the shape of the temperature field after convergence between 

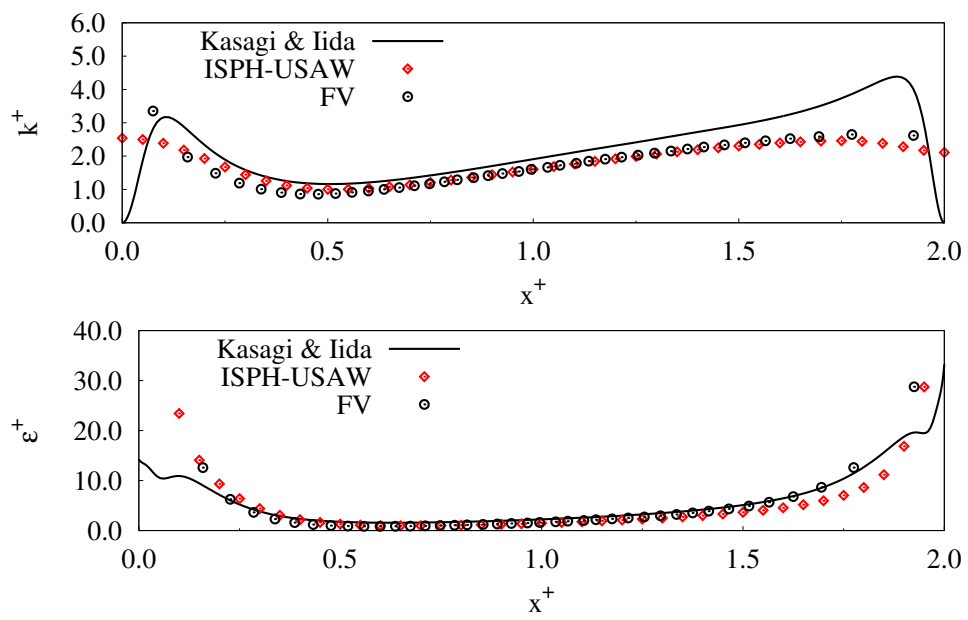

Figure 20: Turbulent plane Poiseuille flow. Profiles of turbulent kinetic energy (top) and dissipation rate (bottom) after convergence. Comparison of ISPHUSAW and FV with DNS results provided by Kasagi \& Iida [18.

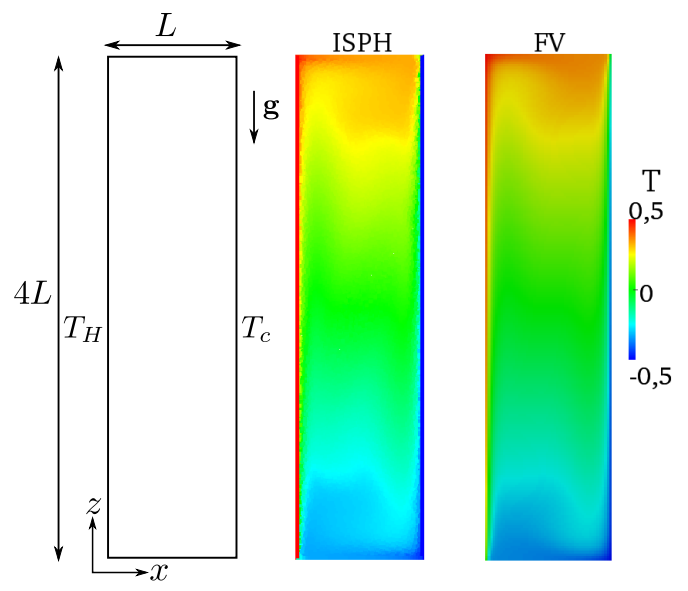

Figure 21: Differentially heated rectangular cavity. Sketch of the case (left) and shape of the temperature field after convergence with ISPH-USAW (middle) and FV (right). 

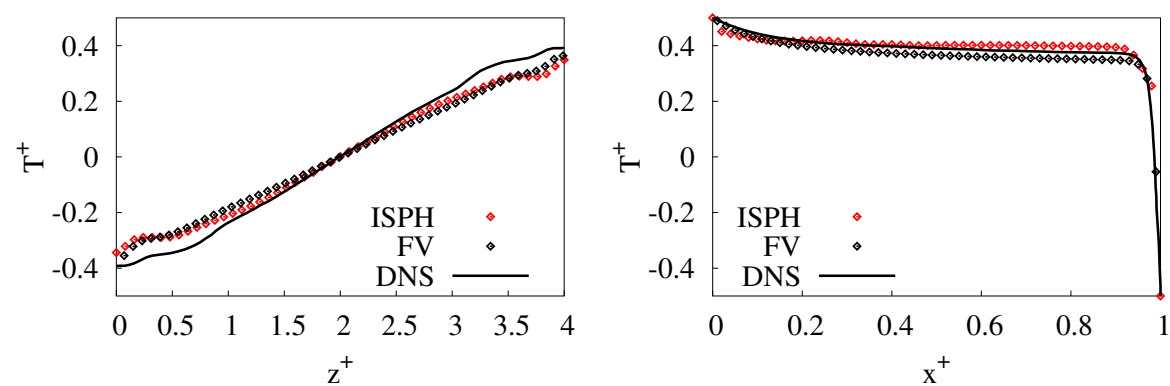

Figure 22: Differentially heated rectangular cavity. Profiles of temperature after convergence along $x^{+}=0.125$ (left) and $z^{+}=1$ (right). Comparison of ISPHUSAW and FV with DNS results provided by Trias et al. [19].

$\mathrm{SPH}$ and FV is provided in Figure 21. Figure 22 shows the temperature profiles along $x^{+}=0.125$ and $z^{+}=1$. A satisfactory agreement is observed between ISPH-USAW and FV. Both methods present reasonable agreement with the DNS results, although some differences are observed, which were expected since a 2D RANS $k-\epsilon$ model is not meant to perfectly reproduce 3D DNS data.

\section{Conclusions}

In this work, a buoyancy model based on the Boussinesq approximation was introduced in an existing ISPH model with a RANS $k-\epsilon$ turbulence closure. The production/destruction term due to buoyancy is taken into account in the $k-\epsilon$ equations. The wall boundary conditions are imposed through the unified semi-analytical technique, which makes it possible to impose arbitrary wall boundary conditions on the fields. In particular, the wall boundary condition imposed on the temperature can be of Dirichlet or Neumann type so as to model isothermal or adiabatic walls, or to impose an arbitrary heat flux through a wall. The results obtained on several configurations of a 2D laminar plane Poiseuille flow show that the wall boundary conditions are properly imposed on the temperature. Several other laminar flows were modelled: a differentially-heated cavity, a differentially heated lid-driven cavity and a lock-exchange. Very good agreement was obtained with mesh-based methods in all cases. The only remaining issue is that the computation of the local Nusselt number is still inaccurate for high temperature gradients, due to a lack of accuracy of the SPH gradient 
operators.

The validation in turbulent mode was done on a $2 \mathrm{D}$ plane Poiseuille flow with two isothermal walls and a differentially-heated rectangular cavity. Comparisons with FV show that the boundary conditions in turbulent mode are satisfactorily imposed in the SPH model, in spite of some differences on $k$ and $\epsilon$ compared to FV close to the wall.

Since buoyancy plays an important part in many industrial and environmental flows, the interest of its modelling is obvious. Modelling this process through a Lagrangian method presents the advantage of avoiding artificial diffusion, which makes SPH an appealing approach for this purpose. In view of this, further work should concern the extension of the present model to $3 \mathrm{D}$, which will require GPU programming.

One should note that additional benefits could be obtained with LES (Large Eddy Simulation). The RANS model used here is relevant for performing simple simulations in the Industry, but fails to predict correlations between momentum and scalar concentration, which can be useful in some specific situations. Since SPH has already been applied to LES simulations with promising results (see e.g. [20, 21, 22, 23]), the present buoyancy model is expected to provide an interesting approach under the LES framework. This would of course avoid using the $k-\epsilon$ model, thus giving a better prediction of heat turbulent fluxes. Such an attempt is let to further investigation.

\section{Acknowledgements}

This work was partly funded by the French Research Agency (CIFRE agreement \# 2011-0264). 


\section{References}

[1] J. N. Reddy and D. K. Gartling. The finite element method in heat transfer and fluid dynamics. CRC press, 2010.

[2] P.J. Oliveira and R. Issa. An improved piso algorithm for the computation of buoyancy-driven flows. Numerical Heat Transfer, Part B: Fundamentals: An International Journal of Computation and Methodology, 40(6):473-493, 2001.

[3] K. Szewc, J. Pozorski, and A. Tanière. Modeling of natural convection with Smoothed Particle Hydrodynamics: Non-Boussinesq formulation. International Journal of Heat and Mass Transfer, 54(23-24):4807-4816, 2011.

[4] V.A. Ghasemi, B. Firoozabadi, and M. Mahdinia. 2D numerical simulation of density currents using the SPH projection method. European Journal of Mechanics - B/Fluids, 38:38-46, 2013.

[5] A. Leroy, D. Violeau, M. Ferrand, and C. Kassiotis. Unified semi-analytical wall boundary conditions applied to 2-D incompressible SPH. Journal of Computational Physics, 261:106-129, March 2014.

[6] W. Rodi. Turbulence models and their applications in hydraulics. In IAHR monograph, Brookfield, Rotterdam, 2000.

[7] V. Guimet and D. Laurence. A linearised turbulent production in the k- $\epsilon$ model for engineering applications. In Proc. Vth International Symposium on Engineering Turbulence Modelling and Measurements, pages 157-166, 2002. Majorqua (Spain).

[8] B.E. Launder and D.B. Spalding. Mathematical models of turbulence. London: Academic Press, 1972.

[9] J. J. Monaghan. Smoothed particle hydrodynamics. Reports on Progress in Physics, 68:1703-1759, 2005.

[10] M. Ferrand, D. R. Laurence, B. D. Rogers, D. Violeau, and C. Kassiotis. Unified semi-analytical wall boundary conditions for inviscid, laminar or turbulent flows in the meshless SPH method. International Journal for Numerical Methods in Fluids, 71:446-472, 2013.

[11] S. Kulasegaram, J. Bonet, R. W. Lewis, and M. Profit. A variational formulation based contact algorithm for rigid boundaries in two-dimensional SPH applications. Computational Mechanics, 33:316-325, 2004.

[12] S.J. Lind, R. Xu, P.K. Stansby, and B.D. Rogers. Incompressible smoothed particle hydrodynamics for free-surface flows: A generalised diffusion-based algorithm for stability and validations for impulsive flows and propagating waves. Journal of Computational Physics, 231(4):1499-1523, 2012.

[13] EDF R\&D. Code_Saturne 3.0.0 Theory Guide, 2013. http://code-saturne. $\mathrm{org} / \mathrm{cms} / \mathrm{sites} / \mathrm{def}$ ault/files/theory-3.0.pdf

[14] H. Wendland. Piecewise polynomial, positive definite and compactly supported radial functions of minimal degree. Advances in Computational Mathematics, 4:389-396, 1995.

[15] F. Archambeau, N. Méchitoua, and M. Sakiz. Code Saturne: a Finite Volume code for the computation of turbulent incompressible flows - industrial applications. International Journal on Finite Volumes, 1:1-62, 2004. 
[16] D.C. Wan, B.S.V. Patnaik, and G.W. Wei. A new benchmark quality solution for the buoyancy-driven cavity by discrete singular convolution. Numerical Heat Transfer, Part B: Fundamentals, 40(3):199-228, 2001.

[17] C. Härtel, E. Meiburg, and F. Necker. Analysis and direct numerical simulation of the flow at a gravity-current head. Part 1. Flow topology and front speed for slip and no-slip boundaries. Journal of Fluid Mechanics, 418:189-212, 2000.

[18] N. Kasagi and O. Iida. Progress in direct numerical simulation of turbulent heat transfer. In Proc. 5th ASME/JSME Joint Thermal Engineering Conference, San Diego, California, 1999.

[19] F.X. Trias, A. Gorobets, M. Soria, and A. Oliva. Direct numerical simulation of a differentially heated cavity of aspect ratio 4 with Rayleigh numbers up to 1011 - Part I: Numerical methods and time-averaged flow. International Journal of Heat and Mass Transfer, 53(4):665-673, 2010.

[20] R. Issa. Numerical assessment of the Smoothed Particle Hydrodynamics gridless method for incompressible flows and its extension to turbulent flows. PhD thesis, University of Manchester, Manchester, 2004.

[21] B. D. Rogers and R. A. Dalrymple. SPH modeling of breaking waves. In Proceedings of the 29th international coastal engineering conference, pages 415-427, 2004.

[22] X. Y. Hu and N. A. Adams. Simulating 3D turbulence with SPH. In Proceedings of the 8th SPHERIC Workshop, pages 377-382, Trondheim, 2013.

[23] A. Mayrhofer, D. Laurence, B. D. Rogers, and D. Violeau. Large eddy simulation with SPH: Mission impossible? In Proc. 9th international SPHERIC workshop, pages 216-222, 2014. 
Table .1: Values of the $k-\epsilon$ model constants [8]

\begin{tabular}{|c|c|c|c|c|c|}
\hline$\kappa$ & $C_{\mu}$ & $C_{\epsilon_{1}}$ & $C_{\epsilon_{2}}$ & $\sigma_{k}$ & $\sigma_{\epsilon}$ \\
\hline 0.41 & 0.09 & 1.44 & 1.92 & 1.0 & 1.3 \\
\hline
\end{tabular}

Table .2: Laminar Poiseuille flow: theoretical solutions for $T^{+}$and $p^{*^{+}}$.

\begin{tabular}{cl}
\hline Case (a): TT & Case (b): QT \\
\hline$T^{+}=\frac{1}{2}\left(1+z^{+}\right)$ & $T^{+}=-N u\left(1+z^{+}\right)$ \\
$p^{*^{+}}=\frac{z^{+2}}{4}-2 \frac{R e}{G r} x^{+}$ & $p^{*^{+}}=z^{+}\left[\frac{1}{2}-N u\left(1+\frac{z^{+}}{2}\right)\right]-\frac{2 R e}{G r} x^{+}$ \\
\hline
\end{tabular}

